\title{
Multiscale Empirical Interpolation for Solving Nonlinear PDEs
}

\author{
Victor M. Calo ${ }^{1,2}$, Yalchin Efendiev ${ }^{1,3}$, Juan Galvis ${ }^{4}$, Mehdi Ghommem $^{1 *}$ \\ ${ }^{1}$ Center for Numerical Porous Media (NumPor) \\ King Abdullah University of Science and Technology (KAUST) \\ Thuwal 23955-6900, Kingdom of Saudi Arabia \\ ${ }^{2}$ Applied Mathematics \& Computational Science and Earth Sciences \& Engineering \\ King Abdullah University of Science and Technology (KAUST) \\ Thuwal 23955-6900, Kingdom of Saudi Arabia \\ ${ }^{3}$ Department of Mathematics \& Institute for Scientific Computation (ISC) \\ Texas A\&M University \\ College Station, Texas, USA \\ ${ }^{4}$ Departamento de Matemáticas, Universidad Nacional de Colombia, \\ Carrera 45 No 26-85 - Edificio Uriel Gutierréz, Bogotá D.C. - Colombia
}

\begin{abstract}
In this paper, we propose a multiscale empirical interpolation method for solving nonlinear multiscale partial differential equations. The proposed method combines empirical interpolation techniques and local multiscale methods, such as the Generalized Multiscale Finite Element Method (GMsFEM). To solve nonlinear equations, the GMsFEM is used to represent the solution on a coarse grid with multiscale basis functions computed offline. Computing the GMsFEM solution involves calculating the system residuals and Jacobians on the fine grid. We use empirical interpolation concepts to evaluate these residuals and Jacobians of the multiscale system with a computational cost which is proportional to the size of the coarse-scale problem rather than the fully-resolved fine scale one. Empirical interpolation method use basis functions which are built by sampling the non-linear function we want to approximate a limited number of times. The coefficients needed for this approximation are computed in the offline stage by inverting an inexpensive linear system.The proposed multiscale empirical interpolation techniques: (1) divide computing the nonlinear function into coarse regions; (2) evaluate contributions of nonlinear functions in each coarse region taking advantage of a reduced-order representation of the solution; and (3) introduce multiscale proper-orthogonal-decomposition techniques to find appropriate interpolation vectors. We demonstrate the effectiveness of the proposed methods on several nonlinear multiscale PDEs that are solved with Newton's methods and fully-implicit time marching schemes. Our numerical
\end{abstract}

*Email address : mehdig@vt.edu 
results show that the proposed methods provide a robust framework for solving nonlinear multiscale PDEs on a coarse grid with bounded error and significant computational cost reduction.

\section{Introduction}

Solving nonlinear Partial Differential Equations (PDEs) with multiple scales and/or high-contrast in media properties is computationally expensive because of the disparity between scales that need to be represented and the inherent nonlinearities. For this reason, coarse-grid computational models are often used. These include Galerkin multiscale finite elements [3, 8, 10, 15-17, 22], mixed multiscale finite element methods [1, 2, 4, 25], the multiscale finite volume method [26], mortar multiscale methods [5, 27], and variational multiscale methods [24]. The coarse-grid models for nonlinear PDEs can be divided into several classes. One of them includes constructing nonlinear operators that allow downscaling from coarse-grid functions to fine-grid functions [18, 19]. Other types of approaches involve designing linear multiscale basis functions (which are constructed using nonlinear PDEs) on the coarse grid and using these basis functions to approximate the solution [11].

One of the challenges in these coarse-grid models is evaluating nonlinear functionals for computing the residual and the Jacobian of the operator. To reduce the computational complexity of these integration (quadrature) procedure, we reinterpret the process as an approximation problem of the resulting nonlinear functionals. Widely used approximation techniques are based on Empirical Interpolation Methods (EIM) [6, 7, 9]. In the offline stage, the nonlinear function is evaluated to yield solution snapshots and interpolation vectors are constructed using the Proper Orthogonal Decomposition (POD) [23]. Evaluating a few components of the nonlinear function allows performing rapid evaluation of the nonlinear function during the online stage with sufficient accuracy [7]. This procedure allows for rapid evaluations of the nonlinear function's response in the online stage. When solving multiscale equations, these empirical interpolation techniques can be expensive because of the large problem sizes. In this paper, we design an efficient multiscale empirical interpolation framework.

Our proposed empirical interpolation techniques are based on multiscale finite element approximations that are often used for solving multiscale problems on coarse grids. The main idea of these methods is to construct local multiscale basis functions for approximating the solution over each coarse patch. Constructing these basis functions uses offline coarse-grid spaces. These spaces are constructed via judicious choices of snapshot spaces and local spectral problems that are motivated by the analysis [11]. The main objective of this paper is to design efficient local multiscale empirical interpolations that can be used in conjunction with Generalized Multiscale 
Finite Element Method (GMsFEM) to solve nonlinear multiscale problems with a computational cost proportional to the number of coarse-scale degrees of freedom rather than the fully resolved mesh.

When GMsFEM is used for nonlinear problems, we evaluate the residual and Jacobian in each Newton iteration which requires fine-grid evaluations and incur a high computational cost. We use POD-based empirical interpolation and follow the Discrete Empirical Interpolation Method (DEIM) introduced in [7]. For this reason, we refer to our method as multiscale DEIM. The main ingredients of the proposed multiscale DEIM are:

- The evaluation of the nonlinear function is performed on the subregions associated with the support of local multiscale basis functions and a global coupling of these local representations is performed.

- In each subregion, an empirical interpolation approximates the nonlinear function which is computed based on a small dimensional multiscale representation of the solution space.

- For multiscale high-contrast problems, interpolation vectors are computed using appropriate spectral problems which involve local multiscale basis functions and ensure convergence of the method. These modified spectral problems are constructed using bounds provided by the numerical analysis of the system.

In this paper, we show that one can design efficient empirical interpolation techniques in conjunction with GMsFEM. We provide error estimates and derive new spectral problems for computing empirical interpolation vectors.

Representative numerical results are presented in the paper. We consider three examples: (1) nonlinear function evaluations using GMsFEM basis functions; (2) steady-state multiscale elliptic equation with nonlinear forcing; and (3) nonlinear multiscale parabolic equations with nonlinear diffusion coefficients. In all examples, we show that, by using a multiscale empirical interpolation, one can obtain an accurate solution approximation at a cost that scales with the coarse-grid size and is independent of the fine-grid size. In particular, a few interpolation modes are needed in all these examples.

The paper is organized as follows. In Section 2, we discuss empirical interpolation methods and local multiscale techniques. In Section 3, we introduce a multiscale empirical interpolation and discuss its convergence. In Section 4, applications of the multiscale empirical interpolation techniques to nonlinear PDEs are studied. In this section, we discuss GMsFEM and the use of multiscale interpolation techniques in Newton methods. Numerical results are presented in Section 5. 


\section{Review of basic concepts}

\subsection{Discrete Empirical interpolation method}

In this paper, we use the Discrete Empirical Interpolation Method (DEIM) [7] for the local approximation of nonlinear functions though other empirical interpolation methods can also be used [6]. DEIM approximates a nonlinear function by a projection constructed using the values of the nonlinear function on a few selected interpolation nodes. We briefly review DEIM following [7]. First, we give some motivation for using DEIM. Let $f(\tau) \in \mathbb{R}^{n}$ denote a nonlinear function, where $\tau$ refers to any control parameter. In reduced-order modeling, the state vector $\tau$ is typically assumed to have a reduced-order representation, i.e., $\tau \in \mathbb{R}^{\widehat{n}}$ can be represented by fewer basis vectors, $\zeta_{1}, \ldots, \zeta_{l}$, where $l \ll \widehat{n}$. In general, $\widehat{n}$ is different from $n$, though we can assume $\widehat{n}=n$. The reduced-order representation of $\tau$ usually leads us to look for a reduced-order representation of the nonlinear functions $f(\tau)$. The procedure of finding the reduced-order representation of $f(\tau)$ consists of two steps. In the first step, we would like to find $m$ basis vectors (where $m$ is much smaller than $n$ ), $\psi_{1}, \ldots, \psi_{m}$, such that $f(\tau)$ can be approximated in the space spanned by these vectors; in practice this can be obtained by selecting a set of values $\tau=\tau_{i}$ and decomposing the set of snapshots $f\left(\tau_{i}\right)$ using POD. The error of this approximation is given by the POD error which approximates of all possible $f\left(\tau_{i}\right)$ 's in the space spanned by $\psi_{1}, \ldots, \psi_{m}$ [7]. In the second step, we identify a reduced-dimensional linear system to find the representation of $f(\tau)$ in this space without involving $n$ operations. In this step, we typically identify $m$ equations to find the coordinates of $f(\tau)$ in the space spanned by $\psi_{1}, \ldots, \psi_{m}$.

In DEIM procedures (see [7]) we assume that for any parameter value of interest $\tau$, a good approximation of $f(\tau)$ is obtained by projecting $f(\tau)$ into a subspace spanned by the basis functions $\Psi=\left(\psi_{1}, \cdots, \psi_{m}\right) \in \mathbb{R}^{n \times m}$ whose linear combinations generate the snapshots obtained by, e.g., forward simulations. We write

$$
f(\tau) \approx \Psi d(\tau) .
$$

To compute the coefficient vector $d$, we select $m$ rows of (1) and invert a reduced system to compute $d(\tau)$. This can be formalized using the matrix $\mathrm{P}$

$$
\mathbf{P}=\left[e_{\wp_{1}}, \cdots, e_{\wp_{m}}\right] \in \mathbb{R}^{n \times m},
$$

where $e_{\wp_{i}}=[0, \cdots, 0,1,0, \cdots, 0]^{T} \in \mathbb{R}^{n}$ is the $\wp_{i}^{\text {th }}$ column of the identity matrix $\mathrm{I}_{n} \in \mathbb{R}^{n \times n}$ for $i=1, \cdots, m$. Multiplying Equation (1) by $\mathrm{P}^{T}$ and assuming that the matrix $\mathrm{P}^{T} \Psi$ is nonsingular, we obtain

$$
f(\tau) \approx \Psi d(\tau)=\Psi\left(\mathrm{P}^{T} \Psi\right)^{-1} \mathrm{P}^{T} f(\tau)
$$


To summarize, approximating the nonlinear function $f(\tau)$, as given by Equation (2), requires the following:

- computing the projection basis $\Psi=\left(\psi_{1}, \cdots, \psi_{m}\right)$.

- identifying the indices $\left\{\wp_{1}, \cdots, \wp_{m}\right\}$.

To determine the projection basis $\Psi=\left(\psi_{1}, \cdots, \psi_{m}\right)$, we collect function evaluations in an $n \times n_{s}$ matrix $\mathrm{F}=\left[f\left(\tau_{1}\right), \cdots, f\left(\tau_{n_{s}}\right)\right]$ and employ the POD technique to select the most energetic modes. This selection uses the eigenvalue decomposition of the square matrix $\mathrm{F}^{T} \mathrm{~F}$ and selects the important modes using the dominant eigenvalues. These modes are used as the projection basis in the approximation given by Equation (1). In Equation (2), the term $\Psi\left(\mathrm{P}^{T} \Psi\right)^{-1} \in \mathbb{R}^{n \times m}$ is computed once and stored. The $d(\tau)$ is computed using the values of the function $f(\tau)$ at $m$ points with the indices $\wp_{1}, \cdots, \wp_{m}$ (identified using the DEIM algorithm). The computational savings are due to the fewer evaluations of $f(\tau)$ that are required. We refer to [7] for further details.

Remark 1 (Inclusion of a priori multiscale information on the POD selection procedure). In this paper, we explore ways to include the information about heterogeneities in the POD selection process. POD selects the dominant eigenpairs of $\left(F^{T} F\right) z_{i}=\lambda_{i} z_{i}$, where $z_{i}$ are coordinates in the space Span $\left\{f\left(\tau_{1}\right), \cdots, f\left(\tau_{n_{s}}\right)\right\}$. In multiscale high-contrast problems, one needs to take into account the heterogeneities when calculating the POD modes. Depending on the application in mind and on a priori information on the space of functions $u$ for which $f(u)$ needs to be computed, it is possible to improve the approximation using different inner products, say, represented by $M_{1}$ and $M_{2}$, and perform a spectral selection based on the eigenvalues of a generalized eigenvalue problem $F^{T} M_{1} F=\lambda F^{T} M_{2} F z$.

\subsection{Local multiscale interpolation}

In multiscale problems, one needs to construct local approximations of the solution using appropriately designed multiscale basis functions. We use GMsFEM, a general local multiscale strategy [11]. These methods construct local multiscale basis functions to approximate the solution over each coarse patch. Constructing these basis functions uses local offline spaces. These spaces are constructed via judicious choices of snapshot spaces and local spectral problems that are motivated by the analysis. Local multiscale basis functions are defined on a coarse grid where each coarse-grid block is a union of fine-grid blocks (see Figure 1 for a schematic representation of the coarse and fine grids).

Next, we discuss the coarse-grid projection operators without going into details. A detailed construction is presented in Section 4.2. Assume that the fine-scale problem has $n_{f}$ degrees of freedom and that the coarse-scale problem has $n_{c}$ degrees of freedom. We design a matrix $\Phi$ of size $n_{f} \times n_{c}$ whose transpose $\Phi^{T}$ of size $n_{c} \times n_{f}$ maps the fine-grid vectors to vectors of coarse 


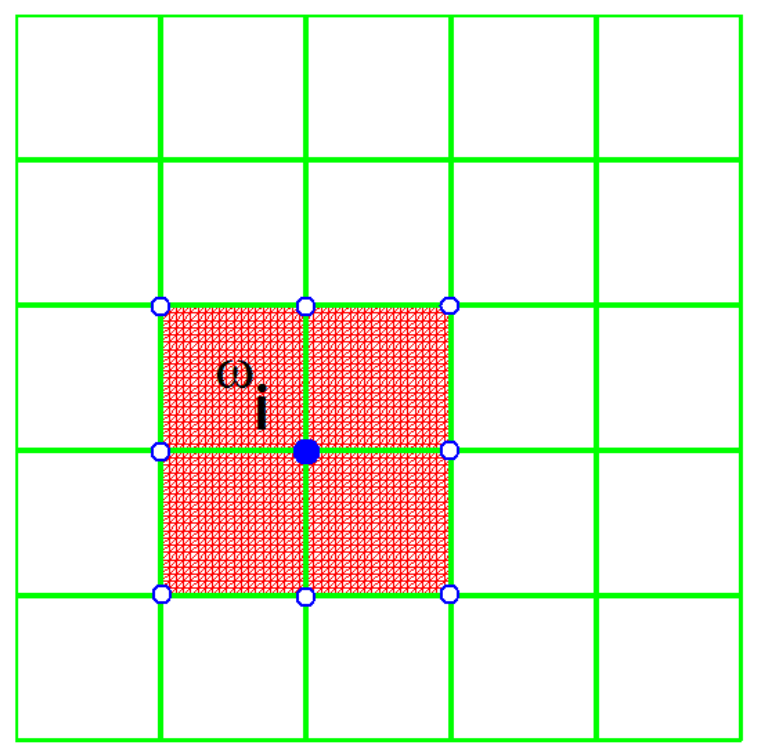

Figure 1: A coarse-grid decomposition of a two-dimensional square domain. We highlight the $i$-th coarse-node (blue circle), its neighborhood $\omega_{i}$ and all other coarse nodes in $\omega_{i}$ (white circles). The fine-mesh is depicted only for coarse-grid blocks forming the highlighted support $\omega_{i}$ of the $i$-th coarse basis.

degrees of freedom (coarse vectors). In this paper we refer to $\Phi$ as downscaling (or coarse-tofine) operator and to $\Phi^{T}$ as upscaling (or fine-to-coarse projection) operator. This is a standard terminology in multiscale and numerical homogenization methods, see [16]. This construction depends on several ingredients, such as the snapshot space and the eigenvalues of the offline and online problems (see Section 4.2 for details on the construction of $\Phi$ using GMsFEM).

Locality is key to the design of the new class of DEIM techniques proposed herein. Each column of $\Phi$ is a multiscale basis function supported locally on a coarse region (see Figure 2 for an example of a basis in a coarse region). One usually has several multiscale basis functions per coarse-grid block. In the design of our multiscale DEIM, we identify multiscale basis functions (columns of $\Phi$ ) which have support in $\omega_{i}$. Only these selected multiscale basis functions are used in obtaining the local DEIM approximation of $f(u)$.

When solving nonlinear PDEs, one writes the residual on the fine grid as

$$
R(u)=0,
$$

where $R(u)$ is the residual of nonlinear PDE and $u$ is the fine-grid solution. For instance, in a nonlinear flow problem we may have a nonlinear partial differential equation (in strong form) given by $R(u)=\alpha(x) \frac{\partial u}{\partial t}-\operatorname{div}\left(\kappa\left(x, u, \mu_{\kappa}\right) \nabla u\right)-g(u, x, \mu)$; see Sections 4.3 and 5 for further 

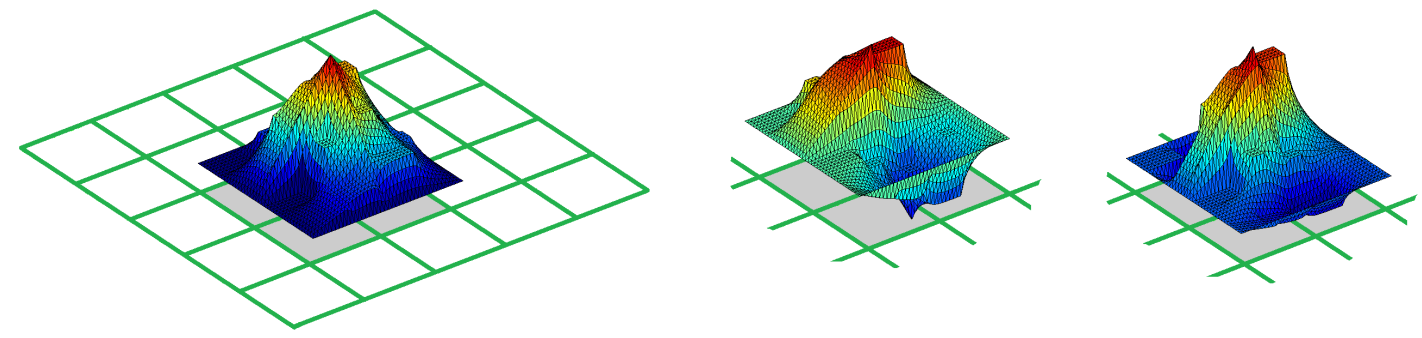

Figure 2: Illustration of three basis functions supported in the highlighted neighborhood.

details and examples. Here, after suitable discretization we can interpret, both $u$ and $R(u)$, as $n_{f^{-}}$ dimensional vectors defined on a fine grid. We use the same symbols for functions and its vector representations in the corresponding discretize problem (e.g., finite element vector representations) since there is no danger of confusion.

Using the projection operator $\Phi$, we project (3) onto the coarse degrees of freedom (noting that $\Phi z$ is an approximation of the fine-grid solution, that is, $u \approx \Phi z$ )

$$
\Phi^{T} R(\Phi z)=0 .
$$

This equation is formulated on the coarse degrees of freedom constructed on the coarse grid; however, computing the residual $R(\Phi z)$ requires fine-grid evaluations. Moreover, computing the Jacobians for each Newton iteration, defined as

$$
J(z)=\nabla_{z} R(\Phi z)
$$

also requires fine-grid evaluations. Here, our main goal is to use the multiscale DEIM to compute $R(\Phi z)$ and $J(z)$ efficiently. In particular, using the multiscale DEIM approximation similar to (1) (with $n=n_{f}$ ), we can write

$$
R(\Phi z) \approx \Psi d(z) .
$$

Consequently from (4) and (5), the residual computation involves

$$
\Phi^{T} \Psi d(z),
$$

which can be efficiently computed by pre-computing $\Phi^{T} \Psi$. A similar procedure can be applied to compute the Jacobian $J(z)$. 


\section{Multiscale Discrete Empirical Interpolation Methods}

\subsection{Algorithm}

We detail the multiscale Discrete Empirical Interpolation Methods (multiscale DEIM). A key idea is that, instead of solving the fine-grid problem (3), we solve the coarse problem (4). An important issue in the solution of the coarse problem (4) is the evaluation of the nonlinear terms. In order to fix ideas, we consider scalar problems and the evaluation of the nonlinear terms of the from $f(u)$ where (the fine-grid finite element function) $u$ is a scalar valued function and $f: \mathbb{R} \rightarrow \mathbb{R}$. The methods are also applicable for vector problems and vector functions $f=\left(f_{1}, f_{2}, \ldots, f_{d}\right)$ for some $d$. In our approach we use the DEIM procedure to efficiently evaluate the nonlinear terms. The following observations are explored and ultimately motivate the design of the multiscale DEIM procedure presented below.

- In applications to multiscale PDEs (where one solves (4) instead of the fine-grid problem (3)) the nonlinear functional $f$ needs to be evaluated with vectors of the form $u=\Phi z$ that are the downscaling of solutions obtained by reduced-order models. Thus, $f(\Phi z)$ needs to be computed in the span of coarse-grid snapshot vectors which has a reduced dimension.

- Due to the fact that multiscale basis functions are supported on a coarse-grid neighborhood, it is sufficient to obtain the DEIM approximation in each coarse-node neighborhood.

- More elaborate spectral selections may be needed to identify the elements of empirical interpolation vectors such that the resulting multiscale DEIM approximation is accurate in adequate norms that depend on physical parameters such as the contrast and small scales.

Each column of $\Phi$ is the vector representation of a (fine-grid) finite element function with local support so we can identify columns of $\Phi$ with locally supported basis functions. Let us introduce the notation $I^{\omega_{i}}$ which represents the set of indexes of coarse basis functions (which correspond to the columns of $\Phi$ ) such that these basis functions have support on $\omega_{i}$ (see Figures 1 and 2). Furthermore, we introduce partition of unity diagonal matrices defined on $\omega_{i}$ denoted by $\mathcal{D}_{i}$ and such that

$$
\sum_{i} \mathcal{D}_{i}=\mathrm{I}_{n}
$$

where $\mathrm{I}_{n}$ is the identity matrix of size $n \times n$. Here, for each node $i$, the $\mathcal{D}_{i}$ is a diagonal matrix with the main diagonal that consists of a partition of unity vector $\chi_{i}$, i.e.,

$$
\mathcal{D}_{i}=\operatorname{diag}\left(\chi_{i}\left(x_{1}\right), \chi_{i}\left(x_{2}\right), \ldots, \chi_{i}\left(x_{n}\right)\right)
$$


where $x_{j}$ are fine-grid nodal points.

Thus $f(u)$ can be written as

$$
f(u)=\sum_{i} \mathcal{D}_{i} f(u),
$$

where $\mathcal{D}_{i} f(u)$ is defined only on the fine-grid nodes of $\omega_{i}$ and recall that $f: \mathbb{R} \rightarrow \mathbb{R}$. Then, only a few basis functions (with indices from $I^{\omega_{i}}$ ) contribute to $\mathcal{D}_{i} f(u)$ since for $u=\Phi z=\sum_{j=1}^{n_{c}} z_{j} \phi_{j}$ and $x \in \omega_{i}$ we have $u(x)=\sum_{j \in I^{\omega_{i}}} z_{j} \phi_{j}(x)$. Thus, we have that

$$
f(\Phi z)=\sum_{i} \mathcal{D}_{i} f(\Phi z)=\sum_{i} \mathcal{D}_{i} f\left(\sum_{j \in I^{\omega_{i}}} z_{j} \phi_{j}\right)
$$

where $\phi_{j}$ are basis vectors ( $j$-th columns of $\left.\Phi\right)$.

Next, in each neighborhood $\omega_{i}$, we can perform an empirical interpolation locally since we can write

$$
\sum_{i} \mathcal{D}_{i} f\left(\sum_{j \in I^{\omega_{i}}} z_{j} \phi_{j}\right)=\sum_{i} \mathcal{D}_{i} f^{\omega_{i}}\left(\sum_{j \in I^{\omega_{i}}} z_{j} \phi_{j}\right)
$$

where for a given $z, f^{\omega_{i}}(z)$ represents the components of $f(z)$ corresponding to nodes in $\omega_{i}$. We abuse notation and identify $f^{\omega_{i}}(z)$ with a full length fine-grid vector with zero entries for components outside $\omega_{i}$. Moreover, for $v=\sum_{j \in I^{\omega_{i}}} z_{j} \phi_{j}, f^{\omega_{i}}(v)$ depends only on the entries of the $\phi_{j}$ in $\omega_{i}$. That is we can write (with slight abuse of notation)

$$
\sum_{i} \mathcal{D}_{i} f^{\omega_{i}}\left(\sum_{j \in I^{\omega_{i}}} z_{j} \phi_{j}\right)=\sum_{i} \mathcal{D}_{i} f^{\omega_{i}}\left(\sum_{j \in I^{\omega_{i}}} z_{j} \phi_{j}^{\omega_{i}}\right)=\sum_{i} \mathcal{D}_{i} f^{\omega_{i}}\left(\Phi^{\omega_{i}} z^{\omega_{i}}\right)
$$

where $\phi_{j}^{\omega_{i}}$ are the components of $\phi_{j}$ in $\omega_{i}, \Phi^{\omega_{i}}$ is the matrix with columns $\phi_{j}^{\omega_{i}}$, and, in general, for a given fine-grid vector $v, v^{\omega_{i}}$ represents a vector containing only entries $v_{j}$ with $j \in I^{\omega_{i}}$.

Therefore, noting that $f^{\omega_{i}}$ depends on a few $z$ 's, we perform an empirical interpolation using DEIM. For each coarse region $\omega_{i}$ we apply DEIM as introduced in Section 2.1 to construct an approximation of the type (1) for the function $\tau \mapsto f^{\omega_{i}}\left(\Phi^{\omega_{i}} \tau\right)$ with $\tau=z^{\omega_{i}}$. According to (1), we obtain $\Psi^{\omega_{i}}$ and $d^{\omega_{i}}$ such that the following approximation holds,

$$
f^{\omega_{i}}\left(\sum_{j \in I^{\omega_{i}}} z_{j} \phi_{j}^{\omega_{i}}\right) \approx \tilde{f}^{\omega_{i}}\left(\sum_{j \in I^{\omega_{i}}} z_{j} \phi_{j}^{\omega_{i}}\right):=\Psi^{\omega_{i}} d^{\omega_{i}}\left(z^{\omega_{i}}\right) .
$$

With this empirical interpolation, we have

$$
f(\Phi z) \approx \tilde{f}(\Phi z):=\sum_{i} \mathcal{D}_{i} \tilde{f}^{\omega_{i}}\left(\sum_{j \in I^{\omega_{i}}} z_{j} \phi_{j}^{\omega_{i}}\right)=\sum_{i} \mathcal{D}_{i} \Psi^{\omega_{i}} d^{\omega_{i}}\left(z^{\omega_{i}}\right) .
$$




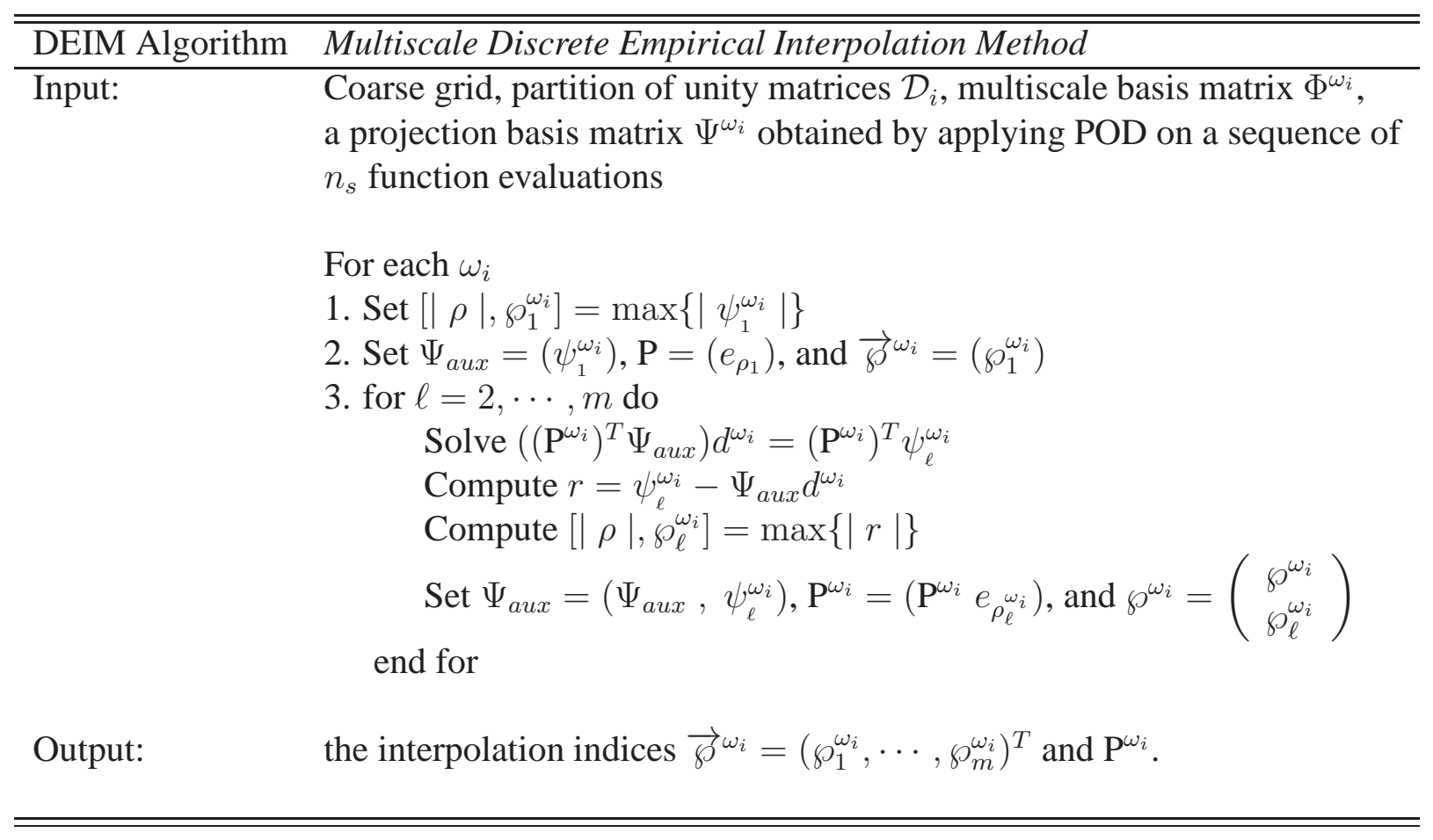

Table 1: Multiscale Discrete Empirical Interpolation Method. This algorithm is used to compute the approximation in (8) for each coarse region. Given $z$ the matrix $\Psi^{\omega}$ is obtained by POD using snapshots $f^{\omega_{i}}\left(\Phi^{\omega} z^{\omega_{i}}\right)$ for some coarse vectors $z$. With the interpolation indices and $\mathrm{P}^{\omega_{i}}$ given by this algorithm we can approximate $f^{\omega_{i}}\left(\Phi^{\omega_{i}} z^{\omega_{i}}\right) \approx$ $\sum_{i} \mathcal{D}_{i} \Psi^{\omega_{i}} d^{\omega_{i}}\left(z^{\omega_{i}}\right)=\sum_{i} \mathcal{D}_{i} \Psi^{\omega_{i}}\left(\left(P^{\omega_{i}}\right)^{T} \Psi^{\omega_{i}}\right)^{-1}\left(P^{\omega_{i}}\right)^{T} f^{\omega_{i}}\left(\Phi^{\omega_{i}} z^{\omega_{i}}\right)$ and observe that $\left(P^{\omega_{i}}\right)^{T} f^{\omega_{i}}\left(\Phi^{\omega_{i}} z^{\omega_{i}}\right)$ requires only the evaluation of the nonlinear function at the interpolation indices.

In simulation, $\mathcal{D}_{i} \Psi^{\omega_{i}}$ can be pre-computed and thus approximating $f(\Phi z)$ can be done at a lower cost.

The algorithm for multiscale DEIM, used to compute the approximation in (8) for each coarse region, is presented in Table 3.1.

\subsection{Analysis}

In the rest of the section, we present the analysis of the multiscale DEIM designed above. In order to simplify the expressions, we use the notation $A \preceq B$ to signify that there is a constant $C$ such that $A \preceq C B$, where this constant $C$ is independent of the vectors involved and of dimension $n$. 
We start by using the definition of $\tilde{f}(\Phi z)$ in (9) to get,

$$
\begin{aligned}
\|f(\Phi z)-\widetilde{f}(\Phi z)\|^{2} & =\left\|\sum_{i} \mathcal{D}_{i} f(\Phi z)-\widetilde{f}(\Phi z)\right\|^{2} \\
& =\left\|\sum_{i} \mathcal{D}_{i}\left(f(\Phi z)-\tilde{f}^{\omega_{i}}\left(\Phi^{\omega_{i}} z^{\omega_{i}}\right)\right)\right\|^{2} \\
& \preceq \sum_{i}\left\|f\left(\Phi^{\omega_{i}} z^{\omega_{i}}\right)-\tilde{f}^{\omega_{i}}\left(\Phi^{\omega_{i}} z^{\omega_{i}}\right)\right\|^{2} \\
& =\sum_{i}\left\|f^{\omega_{i}}\left(\Phi^{\omega_{i}} z^{\omega_{i}}\right)-\Psi^{\omega_{i}} d^{\omega_{i}}\left(z^{\omega_{i}}\right)\right\|_{\omega_{i}}^{2} .
\end{aligned}
$$

Here, we have applied a triangle inequality and the fact that the entries of the diagonal matrices $\mathcal{D}_{i}$ are less than one. The notation $\|\cdot\|_{\omega_{i}}$ corresponds to a vector norm of restricted vectors (to entries associated to nodes in $\omega_{i}$ ). The hidden constant is the maximum number of coarse nodes in $I^{\omega_{i}}$, that is, $\max _{i} \#\left(I^{\omega_{i}}\right)$, where \# is the cardinality of the set. This number is finite, it is independent of the fine-grid parameter $n_{f}$ and depends only on the coarse triangulation configuration.

Following DEIM estimates in [7, Lemma 3.2], we can write

$$
\left\|f^{\omega_{i}}\left(\Phi^{\omega_{i}} z^{\omega_{i}}\right)-\Psi^{\omega_{i}} d^{\omega_{i}}\left(z^{\omega_{i}}\right)\right\|_{\omega_{i}}^{2} \leq\left\|\left(\left(P^{\omega_{i}}\right)^{T} \Psi^{\omega_{i}}\right)^{-1}\right\|^{2}\left\|f^{\omega_{i}}\left(\Phi^{\omega_{i}} z^{\omega_{i}}\right)-\mathcal{P}_{\Psi^{\omega_{i}}} f^{\omega_{i}}\left(\Phi^{\omega_{i}} z^{\omega_{i}}\right)\right\|_{\omega_{i}}^{2} .
$$

Here $\left(\left(P^{\omega_{i}}\right)^{T} \Psi^{\omega_{i}}\right)^{-1}$ is assumed invertible and $\left\|f^{\omega_{i}}\left(\Phi^{\omega_{i}} z^{\omega_{i}}\right)-\mathcal{P}_{\Psi^{\omega_{i}}} f^{\omega_{i}}\left(\Phi^{\omega_{i}} z^{\omega_{i}}\right)\right\|_{\omega_{i}}^{2}$ is the projection of the error onto the space spanned by $\Psi^{\omega_{i}}$ (where $\mathcal{P}_{\Psi^{\omega_{i}}}$ is the orthogonal projection in the vector norm $\|\cdot\|_{\omega_{i}}$ to the space spanned by $\left.\Psi^{\omega_{i}}\right)$.

To estimate $\left\|f^{\omega_{i}}\left(\Phi^{\omega_{i}} z^{\omega_{i}}\right)-\mathcal{P}_{\Psi^{\omega_{i}}} f^{\omega_{i}}\left(\Phi^{\omega_{i}} z^{\omega_{i}}\right)\right\|_{\omega_{i}}^{2}$, we can consider an error estimate that is typical for POD estimates. The error over all snapshots, $\left\{f^{\omega_{i}, s}=f^{\omega_{i}}\left(\Phi^{\omega_{i}} z^{\omega_{i}, s}\right)\right\}_{s=1}^{n^{\omega_{i}}}$, is given by

$$
\sum_{s=1}^{n^{\omega_{i}}}\left\|f^{\omega_{i}, s}-\mathcal{P}_{\Psi^{\omega_{i}}} f^{\omega_{i}, s}\right\|_{\omega_{i}}^{2}=\sum_{s=L_{i}+1}^{n^{\omega_{i}}} \lambda_{s}^{\omega_{i}}
$$

where $n^{\omega_{i}}$ are the number of snapshots in the $\omega_{i}$ region. The $\lambda_{s}^{\omega_{i}}$ are eigenvalues (in decreasing order) of $\Psi^{\omega_{i}}\left(\Psi^{\omega_{i}}\right)^{T}$, and $L_{i}$ is the number of selected eigenvalues whose corresponding eigenvectors are used in the empirical interpolation. This estimate requires that the snapshots used in the above estimate appear in the local POD. One can also show that, for an arbitrary element $b^{\omega_{i}}$ in the span of snapshots, $\operatorname{span}\left\{f^{\omega_{i}, s}\right\}_{s=1}^{n^{\omega_{i}}}$, we have

$$
\left\|b^{\omega_{i}}-\mathcal{P}_{\Psi^{\omega_{i}}} b^{\omega_{i}}\right\|_{\omega_{i}}^{2} \preceq \lambda_{L_{i}+1}^{\omega_{i}}\left\|\left|b^{\omega_{i}} \|\right|_{\omega_{i}}^{2},\right.
$$


where $\left\||\xi \||_{\omega_{i}}^{2}=\sum_{j=1}^{n_{i}^{\omega_{i}}}\left(\lambda_{j}^{\omega_{i}}\right)^{-1}\left(b^{T} \Psi_{j}^{\omega_{i}}\right)^{2}\right.$ with $\Psi_{j}^{\omega_{i}}$ being $j$ th eigenvector. Further, we can write

$$
\begin{aligned}
\sum_{i}\left\|f^{\omega_{i}}\left(\Phi^{\omega_{i}} z^{\omega_{i}}\right)-\Psi^{\omega_{i}} d^{\omega_{i}}\left(z^{\omega_{i}}\right)\right\|_{\omega_{i}}^{2} & \preceq \sum_{i}\left\|\left(\left(P^{\omega_{i}}\right)^{T} \Psi^{\omega_{i}}\right)^{-1}\right\|^{2}\left\|f^{\omega_{i}}\left(\Phi^{\omega_{i}} z^{\omega_{i}}\right)-\mathcal{P}_{\Psi^{\omega_{i}}} f^{\omega_{i}}\left(\Phi^{\omega_{i}} z^{\omega_{i}}\right)\right\|_{\omega_{i}}^{2} \\
& \preceq \sum_{i}\left\|\left(\left(P^{\omega_{i}}\right)^{T} \Psi^{\omega_{i}}\right)^{-1}\right\|^{2}\left\|f^{\omega_{i}}\left(\Phi^{\omega_{i}} z^{\omega_{i}}\right)-\mathcal{P}_{\Psi^{\omega_{i}}} f^{\omega_{i}}\left(\Phi^{\omega_{i}} z^{\omega_{i}}\right)\right\|_{\omega_{i}}^{2} \\
& \preceq \sum_{i}\left\|\left(\left(P^{\omega_{i}}\right)^{T} \Psi^{\omega_{i}}\right)^{-1}\right\|^{2} \lambda_{L_{i}+1}^{\omega_{i}}\left\|\mid f^{\omega_{i}}\left(\Phi^{\omega_{i}} z^{\omega_{i}}\right)\right\| \|_{\omega_{i}}^{2} .
\end{aligned}
$$

One may need a special inner product for multiscale high-contrast equations to ensure an appropriate bound, as discussed in Section 4.4.

\section{Applications to multiscale PDEs}

In this section, we describe the offline-online computational procedure that is used to solve the forward problem on a coarse grid. We elaborate on possible choices for the associated bilinear forms to be used in the coarse space construction. Below we offer a general outline of the multiscale procedure. For details on the constructions and further considerations we refer to [11-14].

1. Offline computations:

1.0. Coarse grid generation.

1.1. Construction of snapshot space used to compute the offline space.

1.2. Construction of a small dimensional offline space by dimensional reduction in the space of local snapshots.

2. Online computations:

2.1. For each input parameter set, compute multiscale basis functions.

2.2. Solution of a coarse-grid problem for given forcing term and boundary conditions.

\subsection{Problem setup}

We consider various non-linear equations of the form

$$
\alpha(x) \frac{\partial u}{\partial t}-\operatorname{div}\left(\kappa\left(x, u, \mu_{\kappa}\right) \nabla u\right)=g(u, x, \mu) \text { in } D,
$$

where $u=0$ on $\partial D$. Here $\mu$ and $\mu_{k}$ represent parameters; see some examples below in Section 5. Performing multiscale simulations requires appropriate local multiscale basis functions. We 
discuss this procedure next. The procedure below identifies local basis functions. Denoting these basis functions by $\phi_{j}$, we seek the solution

$$
u\left(x, t_{n}\right)=\sum_{i} z_{i}^{n} \phi_{i}
$$

that solves (14). We employ an implicit time discretization and use the Newton method to solve the resulting nonlinear system at each time level. In this case, the residual form of problem (14) can be written as

$$
\begin{aligned}
R_{m}\left(z^{n+1}\right) & =\sum_{j} \frac{1}{\Delta t}\left(z_{j}^{n+1}-z_{j}^{n}\right) \int_{D} \alpha(x) \phi_{j} \phi_{m} \\
& +\sum_{j} z_{j}^{n+1} \int_{D} \kappa\left(x, \sum_{l} z_{l}^{n+1} \phi_{l}, \mu_{\kappa}\right) \nabla \phi_{j} \nabla \phi_{m} \\
& -\int_{D} g\left(\sum_{l} z_{l}^{n+1} \phi_{l}, x, \mu\right) \phi_{m}=0,
\end{aligned}
$$

where $z^{n}$ is the solution at the previous time step and $z^{n+1}$ is the value of the solution at the latest time iteration level.

\subsection{Multiscale spatial discretization via GMSFEM}

In this section we review the construction of multiscale (coarse) basis functions. The columns of $\Phi$, introduced in Section 2.2, correspond to vector representation of the multiscale basis functions. In the offline computation, we first construct a snapshot space $V_{\text {snap }}^{\omega_{i}}$. Constructing the snapshot space may involve solving various local problems for different choices of input parameters or different fine-grid representations of the solution in each coarse region. We denote each snapshot vector (listing the solution at each node in the domain) using a single index and create the following matrix

$$
\Phi_{\text {snap }}=\left[\phi_{1}^{\text {snap }}, \ldots, \phi_{M_{\text {snap }}^{\text {snap }}}\right],
$$

where $\phi_{j}^{\text {snap }}$ denotes the snapshots and $M_{\text {snap }}$ denotes the total number of functions to keep in the local snapshot matrix construction.

In order to construct an offline space $V_{\text {off }}$, we perform a dimension reduction process in the space of snapshots using an auxiliary spectral decomposition. The main objective is to use the offline space to efficiently (and accurately) construct a set of multiscale basis functions to be used in the online stage. In each coarse region $\omega_{i}$ separately, we solve the following sequence of problems to construct the offline space. More precisely, we seek for a subspace of the snapshot space to approximate any element of the snapshot space in the appropriate sense which is defined via auxil- 
iary bilinear forms. At the offline stage the bilinear forms are chosen to be parameter-independent, such that there is no need to reconstruct the offline space for each $\nu$ value, where $\nu$ is assumed to be a parameter that represents $u$ and $\mu_{\kappa}$ in $\kappa\left(x, u, \mu_{\kappa}\right)$. For constructing the offline space, we use the average of over the coarse region $\omega_{i}$ in $\kappa(x, \nu)$. Thus, $\nu$ represents both the average of $u$ and $\mu$. We consider the following eigenvalue problem in the space of snapshots:

$$
A^{\text {off }} \Phi_{k}^{\text {off }}=\lambda_{k}^{\text {off }} S^{\text {off }} \Phi_{k}^{\text {off }}
$$

where

$$
\begin{aligned}
& A^{\text {off }}=\left[a_{m n}^{\text {off }}\right]=\int_{\omega_{i}} \bar{\kappa}(x) \nabla \phi_{m}^{\text {snap }} \cdot \nabla \phi_{n}^{\text {snap }}=\Phi_{\text {snap }}^{T} \bar{A} \Phi_{\text {snap }}, \\
& S^{\text {off }}=\left[s_{m n}^{\text {off }}\right]=\int_{\omega_{i}} \widetilde{\kappa}(x) \phi_{m}^{\text {snap }} \phi_{n}^{\text {snap }}=\Phi_{\text {snap }}^{T} \bar{S} \Phi_{\text {snap }} .
\end{aligned}
$$

In the definitions of $A^{\text {off }}$ and $S^{\text {off }}$ above, the coefficient $\bar{\kappa}(x)$ is defined as a parameter-averaged coefficient. The coefficient $\widetilde{\bar{\kappa}}(x)$ can be chosen simply as $\widetilde{\bar{\kappa}}=\bar{\kappa}$ or in more sophisticated manners that include information about multiscale finite element basis functions; we refer to [11] for details and examples. In Equation (18), $\bar{A}$ (similarly for $\bar{S}$ in (19)) denotes a fine-scale matrix, except that parameter-averaged coefficients are used in its construction, and also that $\bar{A}$ is constructed by integrating only on $\omega_{i}$. To generate the offline space, we then choose the smallest $M_{\text {off }}$ eigenvalues from Equation (17) and form the corresponding eigenvectors in the respective space of snapshots by setting $\phi_{k}^{\text {off }}=\sum_{j} \Phi_{k j}^{\text {off }} \phi_{j}^{\text {snap }}$ (for $k=1, \ldots, M_{\text {off }}$ ), where $\Phi_{k j}^{\text {off }}$ are the coordinates of the vector $\Phi_{k}^{\text {off }}$. See $[11,15]$ for further details. We then create the offline matrices

$$
\Phi_{\text {off }}=\left[\phi_{1}^{\text {off }}, \ldots, \phi_{M_{\text {off }}}^{\text {off }}\right]
$$

to be used in the online space construction.

The online coarse space is used within the finite element framework to solve the original global problem, where continuous Galerkin multiscale basis functions are used to compute the global solution. In particular, we seek a subspace of the respective offline space such that it can approximate well any element of the offline space in an appropriate metric.

At the online stage, given a particular value of the parameter, we are required to efficiently find and approximation of the problem for that particular parameter value. We then use the offline spaces to efficiently construct online coarse basis by doing one more spectral selection. The bilinear forms are chosen to be parameter-dependent. The following eigenvalue problems are posed in 
the offline space:

$$
A^{\mathrm{on}}(\nu) \Phi_{k}^{\mathrm{on}}=\lambda_{k}^{\mathrm{on}} S^{\mathrm{on}}(\nu) \Phi_{k}^{\mathrm{on}}
$$

where

$$
\begin{aligned}
A^{\text {on }}(\nu) & =\left[a^{\text {on }}(\nu)_{m n}\right]=\int_{\omega_{i}} \kappa(x, \nu) \nabla \phi_{m}^{\text {off }} \cdot \nabla \phi_{n}^{\text {off }}=\Phi_{\text {off }}^{T} A(\nu) \Phi_{\text {off }}, \\
S^{\text {on }} & =\left[s_{m n}^{\text {on }}\right]=\int_{\omega_{i}} \widetilde{\kappa}(x, \nu) \phi_{m}^{\text {off }} \phi_{n}^{\text {off }}=\Phi_{\text {off }}^{T} S(\nu) \Phi_{\text {off }},
\end{aligned}
$$

and $\kappa(x, \nu)$ and $\widetilde{\kappa}(x, \nu)$ are now parameter dependent, that is, variations in $\mu$ and $u$ are accounted for. As before, the coefficient $\widetilde{\kappa}(x, \nu)$ can be chosen simply as $\widetilde{\kappa}(x, \nu)=\kappa(x, \nu)$ or can include the multiscale finite element basis functions. The choice of $\widetilde{\kappa}$ has implications on the dimensions of the resulting coarse spaces; see [11]. To generate the online space, we then choose the smallest $M_{\text {on }}$ eigenvalues from (20) and form the corresponding eigenvectors in the offline space by setting $\phi_{k}^{\text {on }}=\sum_{j} \Phi_{k j}^{\text {on }} \phi_{j}^{\text {off }}$ (for $k=1, \ldots, M_{\text {on }}$ ), where $\Phi_{k j}^{\text {on }}$ are the coordinates of the vector $\Phi_{k}^{\text {on }}$. If $\kappa(x, u)=k_{0}(x) b(u)$, then one can use the parameter-independent case of GMsFEM. In this case, there is no need to construct the online space (since the online space is the same as the offline space).

For our construction we use $\Phi=\Phi^{\text {on }}$ (see Section 2.2) and from now on, we denote the online space basis functions by $\phi_{i}$.

\subsection{Newton method and Newton-DEIM}

We consider a time-dependent nonlinear flow governed by the following parabolic partial differential equation

$$
\frac{\partial u}{\partial t}-\operatorname{div}(\kappa(x, u, \mu) \nabla u)=h(x) \text { in } D
$$

The finite element discretization of Equation (21) yields a system of ordinary differential equations given by

$$
\mathrm{M} \dot{u}+\mathrm{F}(u)=\mathrm{H} .
$$

Here the mass matrix $M=\left[M_{i j}\right]$ is defined by

$$
\mathrm{M}_{i j}=\int_{D} \phi_{i}^{0} \phi_{j}^{0}
$$


and $\mathrm{F}(u)=\mathrm{A}(u) u$ where $A(u)=\left[A_{i j}(u)\right]$ is given by

$$
\mathrm{A}_{i j}(u)=\int_{D} \kappa(x, u, \mu) \nabla \phi_{i}^{0} \cdot \nabla \phi_{j}^{0}
$$

where $\phi_{i}^{0}$ are piecewise linear basis functions defined on a fine triangulation of $D$. The vector $u$ represents the pressure values at the fine-scale nodes and $\mathrm{H}=\left[H_{i}\right]$ is the right-hand-side vector obtained by discretization and it is given by $H_{i}=\int_{D} \phi_{i}^{0} h$.

In our derivations and simulations, we assume that

$$
\kappa(x, u, \mu)=\sum_{q=1}^{Q} \kappa_{q}(x) b_{q}(u, \mu)
$$

for some $Q$. In general, $\kappa(x, u, \mu)$, can be approximated as in (23) using the offline basis functions (see $[6,11]$ for dealing with the nonlinearity in $u$ within each coarse region). This results in

$$
\mathrm{A}_{i j}(u)=\int_{D} \kappa(x, u, \mu) \nabla \phi_{i}^{0} \cdot \nabla \phi_{j}^{0}=\sum_{q=1}^{Q} \int_{D} b_{q}(u, \mu) \kappa_{q}(x) \nabla \phi_{i}^{0} \cdot \nabla \phi_{j}^{0} .
$$

Now we observe that, for all $x$ in the support of the fine-grid finite element basis function $\phi_{j}^{0}$, which is the union of all elements sharing the vertex $x_{j}$, we can approximate $u(x) \approx u\left(x_{j}\right)$. Using this approximation we get

$$
\mathrm{A}_{i j}(u) \approx \sum_{q=1}^{Q} b_{q}\left(u\left(x_{j}\right), \mu\right) \int_{D} \kappa_{q}(x) \nabla \phi_{i}^{0} \cdot \nabla \phi_{j}^{0}=b_{q}\left(u_{j}, \mu\right) \int_{D} \kappa_{q}(x) \nabla \phi_{i}^{0} \cdot \nabla \phi_{j}^{0}
$$

where $u_{j}=u\left(x_{j}\right)$. If we introduce the matrices $A_{q}$ and the diagonal matrices $\Lambda_{1}^{q}(u, \mu)$ given by

$$
\begin{aligned}
\mathrm{A}_{i j}^{q} & =\int_{D} \kappa_{q} \nabla \phi_{i}^{0} \cdot \nabla \phi_{j}^{0}, \quad q=1, \ldots, Q, \quad \text { and } \\
\Lambda_{1}^{q}(u, \mu) & =\operatorname{diag}\left(b_{q}\left(u_{1}, \mu\right) \quad b_{q}\left(u_{2}, \mu\right) \quad \cdots \quad b_{q}\left(u_{N_{f}}, \mu\right)\right),
\end{aligned}
$$

where $u_{i}=u\left(x_{i}\right)$. We can substitute the function F in (22) by the following approximation,

$$
\mathrm{F}(u, \mu)=\sum_{q=1}^{Q} \mathrm{~A}_{q} \Lambda_{1}^{q}(u, \mu) u
$$

Employing the backward Euler scheme for the time marching process, we obtain

$$
u^{n+1}+\Delta t \mathrm{M}^{-1} \mathrm{~F}\left(u^{n+1}\right)=u^{n}+\Delta t \mathbf{M}^{-1} \mathrm{H},
$$


where $\Delta t$ is the time-step size and the superscript $n$ refers to the temporal level of the solution. We let

$$
\mathrm{R}\left(u^{n+1}\right)=u^{n+1}-u^{n}+\Delta t \mathrm{M}^{-1} \mathrm{~F}\left(u^{n+1}\right)-\Delta t \mathrm{M}^{-1} \mathrm{H}
$$

with derivative

$$
\begin{aligned}
\mathrm{J}\left(u^{n+1}\right)=D \mathrm{R}\left(u^{n+1}\right) & =I+\Delta t \mathrm{M}^{-1} D \mathrm{~F}\left(u^{n+1}\right) \\
& =I+\sum_{q=1}^{Q} \Delta t \mathrm{M}^{-1} \mathrm{~A}_{q} \Lambda_{1}^{q}\left(u^{n+1}\right)+\sum_{q=1}^{Q} \Delta t \mathrm{M}^{-1} \mathrm{~A}_{q} \Lambda_{2}^{q}\left(u^{n+1}\right),
\end{aligned}
$$

where

$$
\Lambda_{2}^{q}(u, \mu)=\operatorname{diag}\left(\begin{array}{llll}
\frac{\partial b_{q}\left(u_{1}, \mu\right)}{\partial u} & \frac{\partial b_{q}\left(u_{2}, \mu\right)}{\partial u} \quad \cdots & \frac{\partial b_{q}\left(u_{N_{f}}, \mu\right)}{\partial u}
\end{array}\right),
$$

and $D$ is the multi-variate gradient operator defined as $D \mathrm{R}(u)=\partial \mathrm{R}_{i} / \partial u_{j}$. The scheme involves, at each time step, the following iterations

$$
\begin{aligned}
\mathrm{J}\left(u_{(k)}^{n+1}\right) \Delta u_{(k)}^{n+1} & =-\left(u_{(k)}^{n+1}-u^{n}+\Delta t \mathrm{M}^{-1} \mathrm{~F}\left(u_{(k)}^{n+1}\right)-\Delta t \mathbf{M}^{-1} \mathrm{H}\right) \\
u_{(k+1)}^{n+1} & =u_{(k)}^{n+1}+\Delta u_{(k)}^{n+1},
\end{aligned}
$$

where the initial guess is $u_{(0)}^{n+1}=u^{n}$ and $k$ is the iteration counter. The above iterations are repeatedly applied until the norm of the residual $R$ is less than a specific tolerance.

In our simulations, we use $Q=1$ (see (23)) for the definition of $Q$ ) as our focus is on localized multiscale interpolation of nonlinear functionals that arise in discretization of multiscale PDEs. With this choice, we do not need to compute the online multiscale space (i.e., the online space is the same as the offline space).

We use the solution expansion given by Equation (15) and employ the multiscale framework to obtain a set of $n_{c}$ ordinary differential equations that constitute a reduced-order model; that is,

$$
\dot{z}=-\left(\Phi^{T} \mathrm{M} \Phi\right)^{-1} \Phi^{T} \mathrm{~F}(\Phi z)+\left(\Phi^{T} \mathrm{M} \Phi\right)^{-1} \Phi^{T} \mathrm{H}
$$

Thus, the original problem with $n_{f}$ degrees of freedom is reduced to a dynamical system with dimension $n_{c}$ where $n_{c} \ll n_{f}$.

The nonlinear term $\left(\Phi^{T} \mathrm{M} \Phi\right)^{-1} \Phi^{T} \mathrm{~F}(\Phi z)$ in the reduced-order model, given by Equation (27), has a computational complexity that depends on the dimension of the full system $n_{f}$. This nonlinear term requires matrix multiplications and full evaluation of the nonlinear function $\mathrm{F}$ at the $n_{f}$-dimensional vector $\Phi z(t)$. As such, solving the reduced system still requires extensive compu- 
tational resources and time. To reduce this computational requirement, we use multiscale DEIM as described in the previous section. In this case, computational savings can be obtained in a forward run of the nonlinear model.

To solve the reduced system, we employ the backward Euler scheme; that is,

$$
z^{n+1}+\Delta t \widetilde{\mathrm{M}}^{-1} \widetilde{\mathrm{F}}\left(z^{n+1}\right)=z^{n}+\Delta t \widetilde{\mathrm{M}}^{-1} \widetilde{\mathrm{H}}
$$

where $\widetilde{\mathrm{M}}=\Phi^{T} \mathrm{M} \Phi, \widetilde{\mathrm{F}}(z)=\Phi^{T} \mathrm{~F}(\Phi z)$, and $\widetilde{\mathrm{H}}=\Phi^{T} \mathrm{H}$. We let

$$
\widetilde{\mathrm{R}}\left(z^{n+1}\right)=z^{n+1}-z^{n}+\Delta t \widetilde{\mathrm{M}}^{-1} \widetilde{\mathrm{F}}\left(z^{n+1}\right)-\Delta t \widetilde{\mathrm{M}}^{-1} \widetilde{\mathrm{H}}
$$

with derivative

$$
\begin{aligned}
\widetilde{\mathbf{J}}\left(z^{n+1}\right)=D \widetilde{\mathrm{R}}\left(z^{n+1}\right) & =I+\Delta t \widetilde{\mathrm{M}}^{-1} D \widetilde{\mathrm{F}}\left(z^{n+1}\right) \\
& =I+\sum_{q=1}^{Q} \Delta t \widetilde{\mathrm{M}}^{-1} \Phi^{T} \mathrm{~A}_{q} \Lambda_{1}^{q}\left(\Phi z^{n+1}\right) \Phi+\sum_{q=1}^{Q} \Delta t \widetilde{\mathrm{M}}^{-1} \Phi^{T} \mathrm{~A}_{q} \Lambda_{2}^{q}\left(\Phi z^{n+1}\right) \Phi .
\end{aligned}
$$

The scheme involves, at each time step, the following iterations

$$
\begin{aligned}
\widetilde{\mathbf{J}}\left(z_{(k)}^{n+1}\right) \Delta z_{(k)}^{n+1} & =-\left(z_{(k)}^{n+1}-z^{n}+\Delta t \widetilde{\mathbf{M}}^{-1} \widetilde{\mathrm{F}}\left(z_{(k)}^{n+1}\right)-\Delta t \widetilde{\mathbf{M}}^{-1} \widetilde{\mathrm{H}}\right) \\
z_{(k+1)}^{n+1} & =z_{(k)}^{n+1}+\Delta z_{(k)}^{n+1},
\end{aligned}
$$

where the initial guess is $z_{(0)}^{n+1}=z^{n}$. The above iterations are repeated until $\left\|\Delta z_{(k)}^{n+1}\right\|$ is less than a specific tolerance. We use multiscale DEIM as detailed in Section 3 to approximate the nonlinear functions that appear in the residual $\widetilde{R}$ and the Jacobian $\widetilde{J}$ and, therefore, reduce the number of function evaluations.

\subsection{The use of multiscale POD for DEIM}

When designing empirical interpolation methods for high-contrast problems, it is important to take into account heterogeneities and perform an interpolation using special weighted norms. These norms are derived based on the analysis discussed next.

Consider the approximation of

$$
\sum_{i} c_{i} \int_{D} \kappa(x) b\left(\sum_{l} z_{l} \phi_{l}\right) \nabla \phi_{i} \nabla \phi_{j}
$$

that appears in the residual function (29). For multiscale DEIM, we split this integral using partition of unity matrices as discussed earlier in (7) and re-write it for the discretization of the stiffness 
matrix. We introduce the partition of unity $\left\{\chi^{\omega_{k}}\right\}$ subordinated to the coarse regions $\left\{\omega_{k}\right\}$. The relation with the partition of unity matrices $\mathcal{D}_{k}$ is that the action of $\mathcal{D}_{k}$ is the multiplication of the corresponding finite element function by the partition of unity function $\chi^{\omega_{k}}$. We obtain,

$$
\begin{aligned}
\sum_{i} c_{i} \int_{D} \kappa(x) b\left(\sum_{l} z_{l} \phi_{l}\right) \nabla \phi_{i} \nabla \phi_{j} & =\sum_{i, k} c_{i} \int_{\omega_{k}} \chi^{\omega_{k}}(x) \kappa(x) b\left(\sum_{l} z_{l} \phi_{l}^{\omega_{k}}\right) \nabla \phi_{i}^{\omega_{k}} \nabla \phi_{j}^{\omega_{k}} \\
& =\sum_{i, k} c_{i} \int_{\omega_{k}} \chi^{\omega_{k}}(x) \kappa(x) b\left(\sum_{l \in I^{\omega_{k}}} z_{l} \phi_{l}^{\omega_{k}}\right) \nabla \phi_{i}^{\omega_{k}} \nabla \phi_{j}^{\omega_{k}} .
\end{aligned}
$$

The interpolation for $b\left(\sum_{l \in I^{\omega_{k}}} z_{l} \phi_{l}^{\omega_{k}}\right)$ is performed in the region $\omega_{k}$. Each term in the sum (33) can be written as

$$
\int_{\omega_{k}} m_{i j}^{\omega_{k}}(x) b\left(\sum_{l \in I^{\omega_{k}}} z_{l} \phi_{l}^{\omega_{k}}\right)
$$

where $m_{i j}^{\omega_{k}}(x)=\chi^{\omega_{k}}(x) \kappa(x) \nabla \phi_{i}^{\omega_{k}} \nabla \phi_{j}^{\omega_{k}}$.

To evaluate (34), one needs to take into account the weight $m_{i j}^{\omega_{k}}(x)$ for high-contrast multiscale problems, where $m_{i j}^{\omega_{k}}(x)$ can have very high values in some subregions within $\omega_{k}$. Otherwise, the accuracy of the method substantially deteriorates. However, the weighting function is not uniquely defined and depends on particular basis functions that are involved in the integration. We propose the use of a weight function that is computed by summing over all indices $i, j$ (which provides an upper bound for every $m_{i j}^{\omega_{k}}(x)$ ) and using only a few dominant modes. For high-contrast problems, $m_{i j}^{\omega_{k}}(x)$ is very high in the regions of high conductivity and this high value can be estimated using only the first basis function. We propose using

$$
\widetilde{m}^{\omega_{k}}=\sum_{i} \kappa(x)\left|\nabla \phi_{1}^{\omega_{i}}\right|^{2}
$$

which is defined in the entire domain. Here, $\phi_{1}^{\omega_{k}}$ is the first basis function (associated to the first dominant mode in the online GMsFEM selection procedure). One can also use additional basis functions to construct the weight $\widetilde{m}^{\omega_{k}}$. For implementing this procedure, we need to define $\Psi^{\omega_{i}}$ in (8) using weighted POD modes. This can simply be done using the matrix corresponding to the mass matrix $\widetilde{m}^{\omega_{k}}$ as $M_{1}$ in Remark 1 ( $M_{2}$ is the identity matrix of appropriate size).

Using the above argument, one can write the error in the residuals of multiscale DEIM $\widetilde{R}^{D}$ and $R(z)$.

$$
R(z)-\widetilde{R}^{D}(z)=\sum_{i, k} c_{i} \int_{\omega_{k}} \chi^{\omega_{k}}(x) \kappa(x)\left(b\left(\sum_{l \in I^{\omega_{k}}} z_{l} \phi_{l}^{\omega_{k}}\right)-\sum_{l} d_{l}(z) \psi_{l}^{\omega_{k}}\right) \nabla \phi_{i}^{\omega_{k}} \nabla \phi_{j}^{\omega_{k}} .
$$


Using an appropriate weight function in POD, we can control the error $R(z)-\widetilde{R}^{D}(z)$ independently of the physical parameters such as the contrast and small scales. This error can be related, to the error between the solution obtained using multiscale DEIM and the solution obtained without DEIM. If a standard (instead of multiscale) POD is used for the selection of empirical modes, then the error depends on the contrast and, in particular, the error is proportional to the contrast. Similarly, the error in the Jacobians corresponding to multiscale DEIM and without DEIM can be controlled independently of the contrast if we choose the weighted POD modes properly. Large errors in the Jacobians may lead to a poor convergence of the Newton method as its convergence rate is related to the quality of approximating the inverse of the Jacobians. We have observed this in our numerical simulations.

The weighted POD procedure allows minimizing the residual error between the snapshots of $b$ 's (nonlinear function) and their projections in the weighted norm. Consequently, the residual is mainly minimized in the high-conductivity regions which are important flow carriers.

\section{Representative numerical experiments}

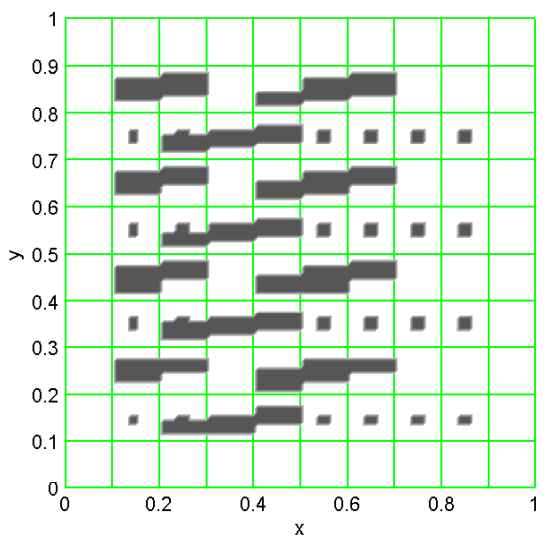

(a) Case I

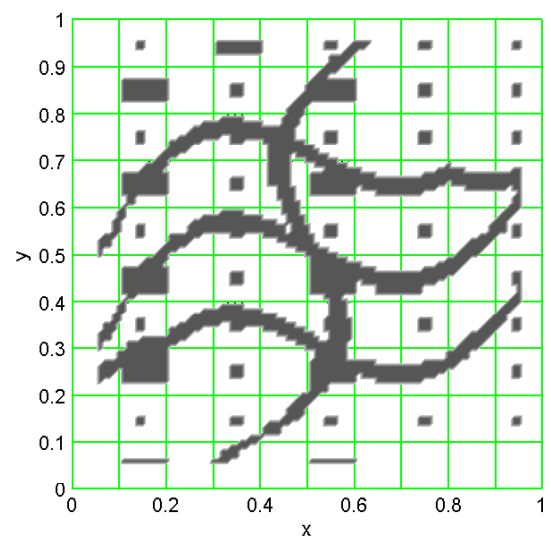

(b) Case II

Figure 3: Different permeability fields considered in the numerical experiments. Each permeability field has a different structures that model high conductivity channels within a homogeneous domain. The minimum (background) conductivity is taken to be $\kappa_{\min }=1$, and the high-conductivity (gray regions) with value of $\kappa_{\max }=10^{\eta}(\eta=4$ or 6$)$.

In this section, we present numerical examples to illustrate the applicability of the multiscale empirical interpolation method for solving nonlinear multiscale partial differential equations. Before presenting the individual examples, we review the computational domain used in constructing the GMsFEM basis functions. This computation is performed during the offline stage. We discretize with finite elements a nonlinear PDE posed on the computational domain $D=[0,1] \times[0,1]$. 
For constructing the coarse grid, we divide $[0,1] \times[0,1]$ into $10 \times 10$ squares. Each square is divided further into $10 \times 10$ squares each of which is divided into two triangles. Thus, the discretization parameters are 1/100 for the fine-grid mesh and 1/10 for the coarse-mesh. The fine-scale finite element vectors introduced in this section are defined on this fine grid. The fine-grid representation of a coarse-scale vector $z$ is given by $\Phi z$, which is a fine-grid vector.

Using the two grids, we construct the GMsFEM coarse space as described in Section 4. In the simulations, we consider two different permeability fields. These permeability fields, denoted by $\kappa$, contain channels of high conductivity and are shown in Figure 3. This figure shows fields with different structure that model high-conductivity channels within a homogeneous domain. The minimum conductivity value for this case is taken to be $\kappa_{\min }=1$, and the high conductivity varies from channel to channel with a maximum value of $\kappa_{\max }=10^{\eta}(\eta=4$ or 6$)$. Both permeability fields are represented by the fine mesh.

\subsection{Nonlinear functions}

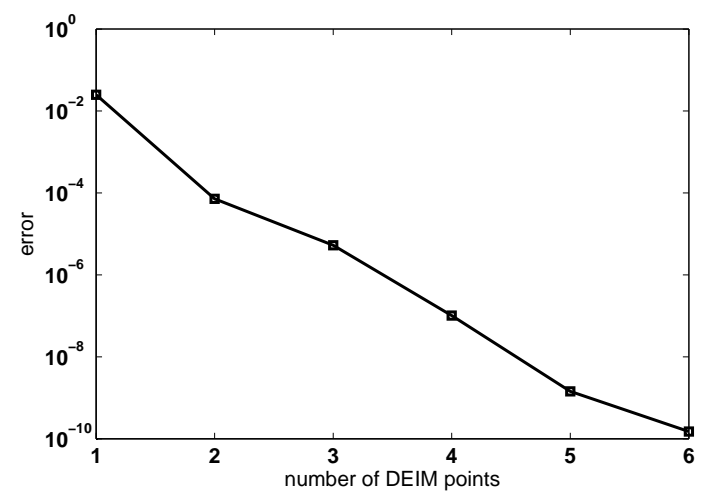

(a) $F_{1}$

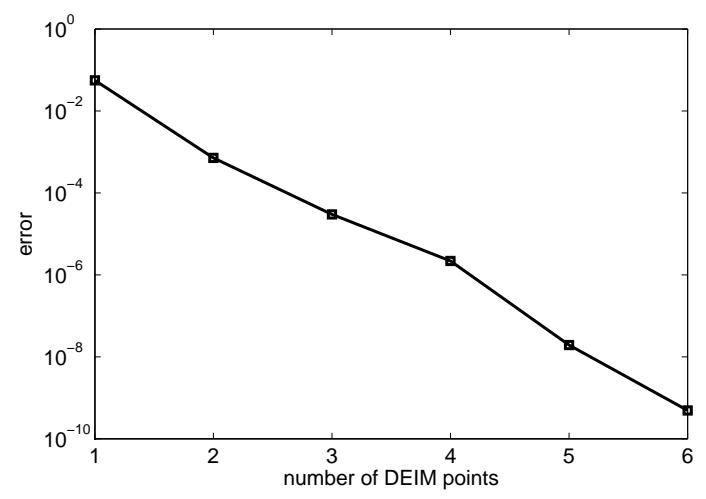

(b) $F_{2}$

Figure 4: Variations of the relative error between the approximate and the original nonlinear functions with the number of DEIM points.

To demonstrate the applicability of the multiscale empirical interpolation method in approximating nonlinear functions when using the multiscale framework, we consider two parametrized functions $F_{1}: \mathbb{R}^{n_{f}} \times[0,1] \mapsto \mathbb{R}^{n_{f}}$ and $F_{2}: \mathbb{R}^{n_{f}} \times[0,1] \mapsto \mathbb{R}^{n_{f}}$ given by

$$
\begin{aligned}
& F_{1}(\Phi z ; \mu)=(\sin (2 \pi \mu \Phi z) \cos (2 \pi \mu \Phi z))^{2} e^{-2 \pi \mu \Phi z} \\
& F_{2}(\Phi z ; \mu)=\frac{1}{1+\sin (2 \pi \mu \Phi z)},
\end{aligned}
$$


where $\mu \in[0,1]$. We follow the multiscale DEIM approach described in Section 3 to approximate the nonlinear function on the fine grid while evaluating at few selected points.

To check the capability of the multiscale DEIM to properly approximate the nonlinear function, we compute the relative error as the $\mathrm{L}_{2}$-norm of the difference between the original and approximate functions: i.e.,

$$
\|E\|_{2}=\frac{\|F-\widetilde{F}\|_{2}}{\|\widetilde{F}\|_{2}},
$$

where $\widetilde{F}$ is obtained from the DEIM approximation. We consider $\Phi z$ as a multiscale solution of the elliptic problem obtained from a fine mesh of dimension 10201 (i.e., $N_{x}=N_{y}=n_{x}=$ $\left.n_{y}=10\right)$ by solving $-\operatorname{div}(\kappa \nabla u)=1$ in $D$ and $u=0$ on $\partial D$, where $\kappa$ is defined as in Case I with $\eta=4$ (see Figure 3). In Figure 4, we plot the relative error variations with the number of DEIM points. As expected, the error decreases as the number of DEIM points is increased. For instance, the relative error $\|E\|_{2}$ is equal to $7.1810^{-3}$ and $7.1410^{-3}$ when using only 2 DEIM points per region to approximate the nonlinear functions $F_{1}$ and $F_{2}$, respectively, defined on a fine mesh of dimension 10201. These results show the capability of the multiscale DEIM to reproduce the fine-scale representation of the nonlinear function while gaining in terms of computational cost through evaluating at only a few selected points.

In Figure 5, we compare the approximate functions obtained from DEIM using two points per region against the original function of dimension $n_{f}=10201$. The good agreement observed between the two sets of data shows the capability of DEIM to approximate the nonlinear function using a few selected points per region.

\subsection{Nonlinear steady PDE}

In this section, we consider a steady non-linear elliptic equation of the form

$$
\operatorname{div}(\kappa(x) \nabla u)=g(u, x, \mu) \text { in } D
$$

where

$$
g(u, x, \mu)=(1+\sin (2 \pi \mu u)) e^{-2 \pi \mu u} .
$$

We consider permeability fields $\kappa$ that contain high-conductivity channels as shown in Figure 3. We use GMsFEM with Newton's method to discretize and solve Equation (38) and employ the multiscale DEIM to approximate the nonlinear forcing term. 


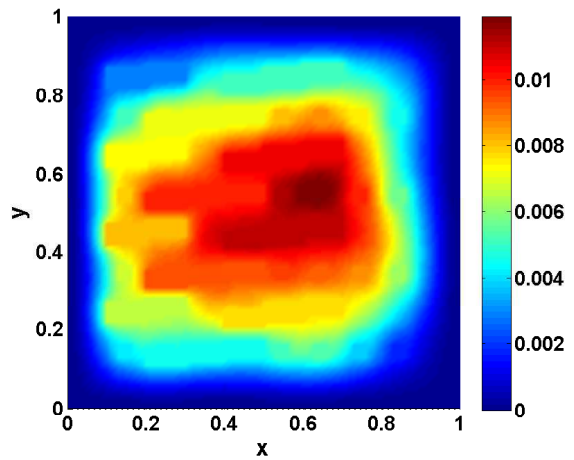

(a) $F_{1}$ - exact function

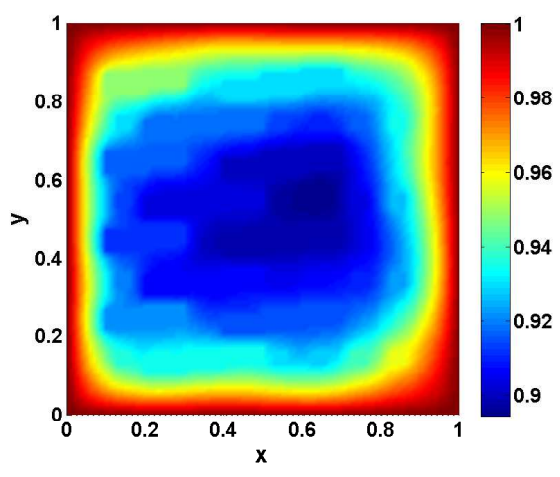

(c) $F_{2}$ - exact function

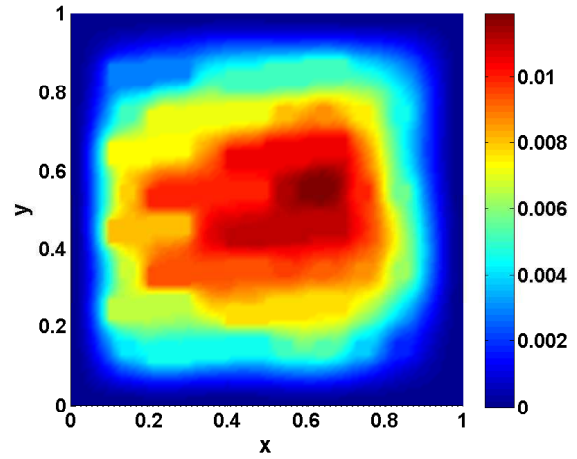

(b) $\widetilde{F}_{1}$ - approximate function

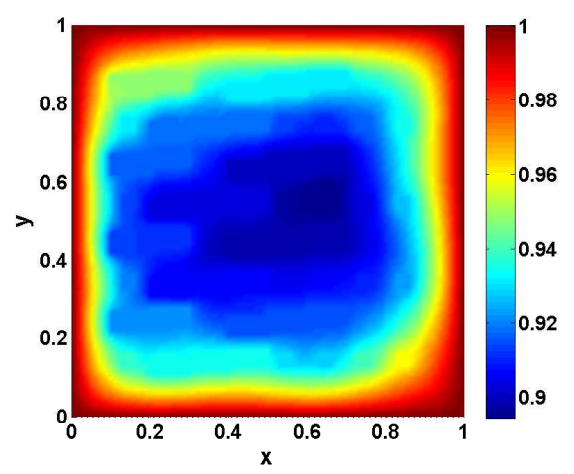

(d) $\widetilde{F}_{2}$ - approximate function

Figure 5: Comparison of the DEIM approximation with the original nonlinear function of dimension $n_{f}=10201$.

We define the relative energy error as

$$
\|E\|_{\mathrm{A}}=\sqrt{\frac{(u-\widetilde{u})^{T} \mathrm{~A}(u-\widetilde{u})}{u^{T} \mathrm{~A} u}}
$$

where A is the fine-scale stiffness matrix that corresponds to (38). The errors are plotted in Figure 6 with respect to the number of DEIM points. Clearly, the use of a few DEIM points yields good approximation of the nonlinear forcing term and, consequently, the nonlinear PDE solution. Our numerical experiments show that the error does not depend on the contrast and it decreases as we increase the number of DEIM points. We refer to $[15,20,21]$ for theoretical results on the error analysis of high-contrast problems.

For illustration purposes, in Figure 7, we show the approximate solutions obtained from the multiscale DEIM approach using two DEIM points per coarse region. The black dots denote the location of the DEIM points. These DEIM points show high absolute values of the modes representing the forcing terms in (38). 


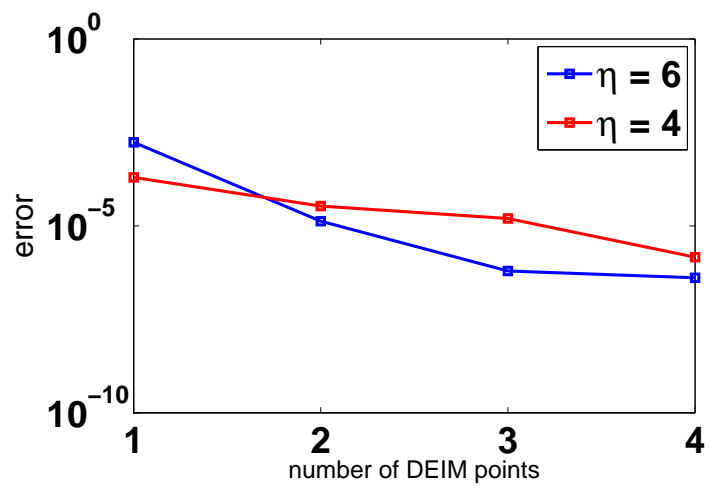

(a) Case I

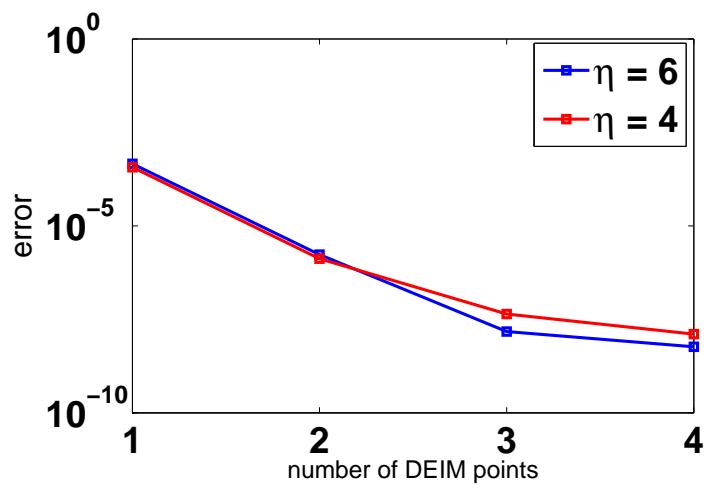

(b) Case II

Figure 6: Variations of the relative energy error between the multiscale and multiscale DEIM solutions with the number of DEIM points.

\subsection{Nonlinear unsteady PDE}

As an example of a nonlinear unsteady problem, we consider the following time-dependent parabolic equation

$$
\frac{\partial u}{\partial t}-\operatorname{div}(\kappa(x ; u, \mu) \nabla u)=h(x) \text { in } D
$$

where

$$
\kappa(x ; u, \mu)=\kappa_{q}(x) b_{q}(u, \mu), \quad b_{q}(u, \mu)=e^{\mu u}, \quad h(x)=1+\sin \left(2 \pi x_{1}\right) \sin \left(2 \pi x_{2}\right),
$$

and the structure of the permeability fields $\kappa_{q}$ is shown in Figure 3. We employ the GMsFEM for space discretization and the Newton method to solve the nonlinear algebraic system at each time step as detailed in Section 4.3. Furthermore, we use multiscale DEIM, described in Section 3, to approximate the nonlinear functions and use multiscale POD (see Section 4.4) to identify the modes in the empirical interpolation. Without multiscale POD, the Newton method may converge slowly or may not converge when using $l_{2}$-inner-product-based POD [23]. In Figure 8, we plot the variation of the relative energy error between the multiscale and multiscale DEIM solutions with the number of DEIM points for the two configurations of the permeability field. The results are obtained for $\mu=10$. Multiscale DEIM approximates well the multiscale solutions as indicated by the small error values while significantly reducing the number of function evaluations.

In Figure 9, we show the approximate solutions obtained from the multiscale DEIM approach using two DEIM points per region. The black dots denote the location of the DEIM points.

We investigate the robustness of the multiscale DEIM approach with respect to moderate vari- 


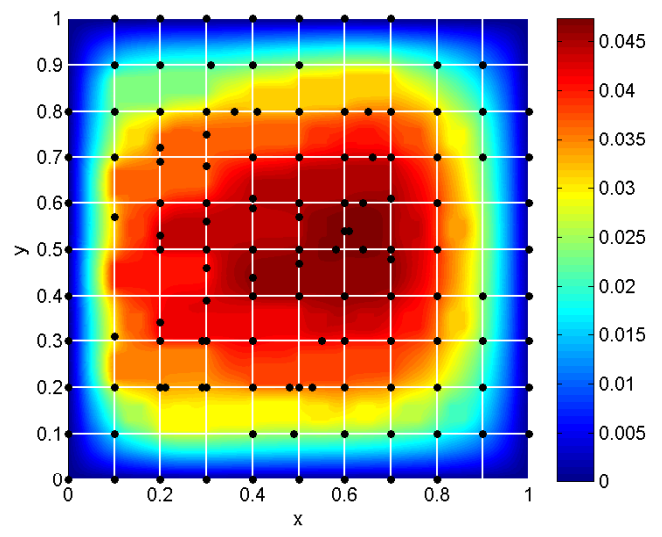

(a) Case I

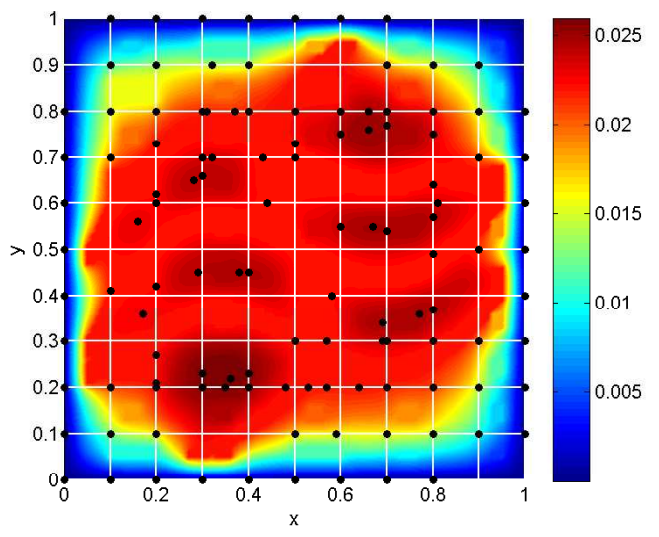

(b) Case II

Figure 7: Coarse grid and approximated solutions obtained from the multiscale DEIM approach using two DEIM points per region. Black dots denote the location of the DEIM points.

ations in the nonlinearity of the permeability field. As such, we run the forward problem while uniformly varying the value of the parameter $\mu$ of the nonlinear function $b_{q}$ over the interval $[5,10]$ with a step equal to one and store the snapshots of the nonlinear functions for each case. Then, we use all snapshots to compute the DEIM points and the index matrices of each region as described in Section 3. Next, we consider a nonlinear function $b_{q}(u, \mu)=e^{\mu u}$ with $\mu=8.5$ and show in Figure 10 the relative energy error variations with the number of DEIM points. Note that $\mu=8.5$ is not among the samples considered to compute the global DEIM points. Using a few DEIM points yields a good approximation. We mention that the simulation time of the final reduced order model is about $4 \%$ of the computational time of running the fine-grid model. For instance, the relative energy error $\|E\|_{\mathrm{A}}$ is equal to $4.9610^{-2}$ and $1.0510^{-2}$ when using only three DEIM points per region to approximate the nonlinear functions that appear in the residual $\widetilde{R}$ and the Jacobian $\widetilde{J}$ for the two configurations of the permeability field. Considering more DEIM points results in smaller error values. These results demonstrate the robustness of the model reduction approach based on combining the multiscale framework and DEIM. We have also tested this approach with different right-hand-sides and observed similar results.

\section{Conclusions}

In this paper, we propose a multiscale empirical interpolation for solving nonlinear multiscale partial differential equations. The proposed method uses the Generalized Multiscale Finite Element Method (GMsFEM) which constructs multiscale basis functions on a coarse grid to solve the 


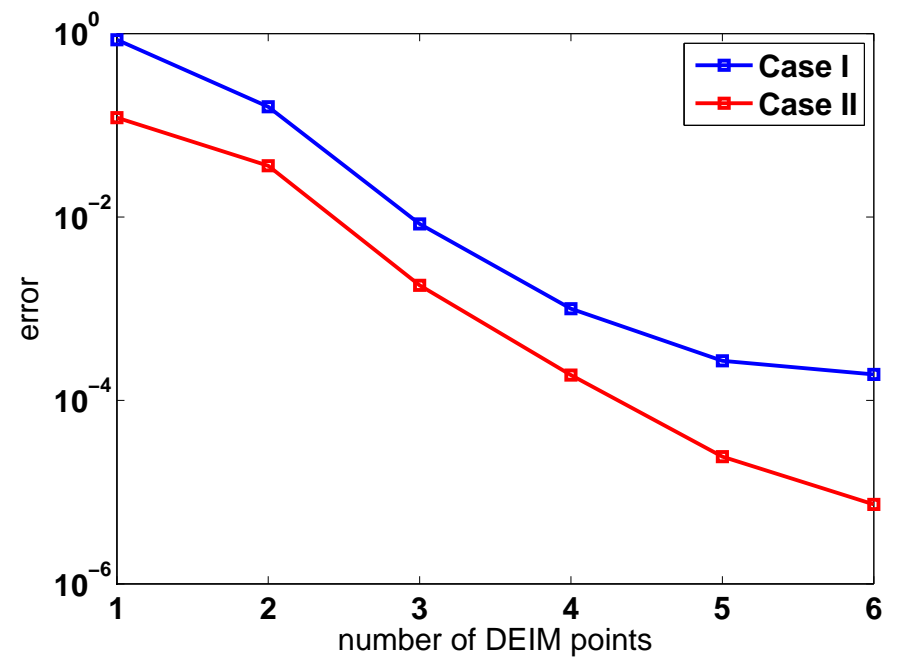

Figure 8: Variations of the relative energy error between the multiscale and multiscale DEIM solutions with the number of DEIM points.

nonlinear problem. To approximate inexpensively nonlinear functions that arise in the residual and Jacobians, we design multiscale empirical interpolation techniques that use empirical modes constructed based on local approximations of the nonlinear functions using weighted POD techniques. The proposed multiscale empirical interpolation techniques (1) divide the computation of the nonlinear function into coarse regions, (2) evaluate the contributions of the nonlinear functions in each coarse region taking advantage of a reduced-order solution representation, and (3) introduce multiscale Proper Orthogonal Decomposition techniques to find appropriate interpolation vectors. We demonstrate the applicability of the proposed methods on several examples of nonlinear multiscale PDEs that are solved with Newton methods. Our numerical results show that the proposed methods provide an accurate and robust framework for solving nonlinear multiscale PDEs on coarse grids while providing significant computational cost savings..

\section{References}

[1] J.E. Aarnes. On the use of a mixed multiscale finite element method for greater flexibility and increased speed or improved accuracy in reservoir simulation. SIAM J. Multiscale Modeling and Simulation, 2:421-439, 2004.

[2] J.E. Aarnes and Y. Efendiev. Mixed multiscale finite element for stochastic porous media flows. SIAM J. Sci. Comput., 30 (5):2319-2339, 2008. 


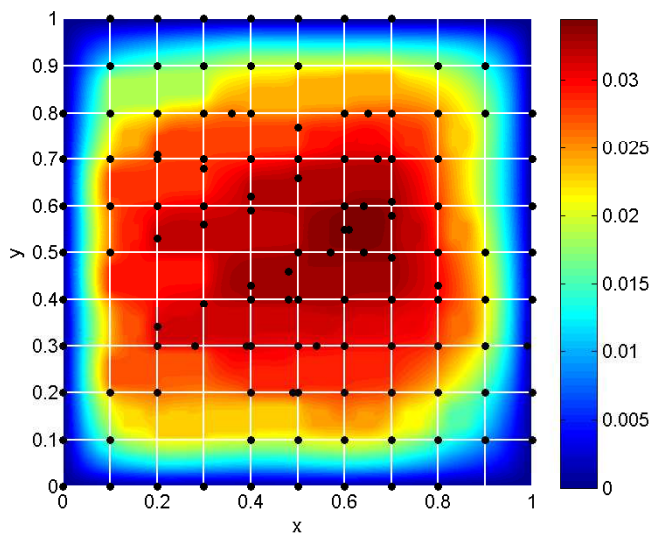

(a) Case I

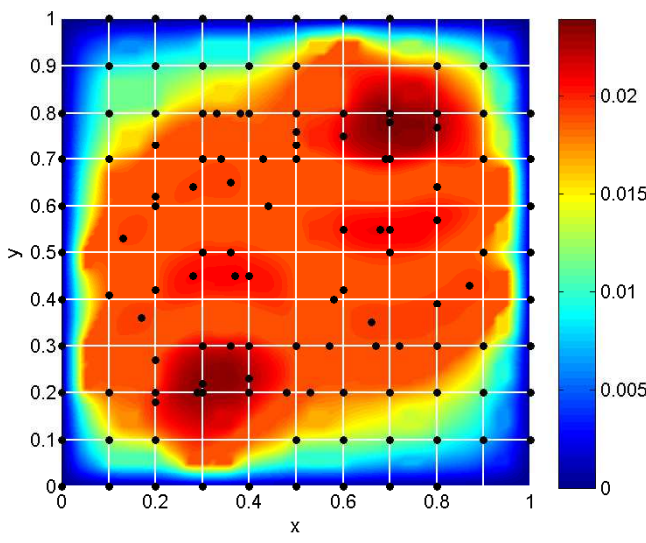

(b) Case II

Figure 9: Approximate solutions obtained from the multiscale DEIM approach using two DEIM points per region. Black dots denote the location of the DEIM points.

[3] T. Arbogast. Analysis of a two-scale, locally conservative subgrid upscaling for elliptic problems. SIAM J. Numer. Anal., 42(2):576-598 (electronic), 2004.

[4] T. Arbogast and K.J. Boyd. Subgrid upscaling and mixed multiscale finite elements. SIAM J. Numer. Anal., 44(3):1150-1171 (electronic), 2006.

[5] T. Arbogast, G. Pencheva, M.F. Wheeler, and I. Yotov. A multiscale mortar mixed finite element method. Multiscale Model. Simul., 6(1):319-346, 2007.

[6] Maxime Barrault, Yvon Maday, Ngoc Cuong Nguyen, and Anthony T. Patera. An 'empirical interpolation' method: application to efficient reduced-basis discretization of partial differential equations. C. R. Math. Acad. Sci. Paris, 339(9):667-672, 2004.

[7] S. Chaturantabut and D.C. Sorensen. Nonlinear model reduction via discrete empirical interpolation. SIAM J. Sci. Comput., 32(5):2737-2764, 2010.

[8] C.-C. Chu, I. G. Graham, and T.-Y. Hou. A new multiscale finite element method for highcontrast elliptic interface problems. Math. Comp., 79(272):1915-1955, 2010.

[9] Martin Drohmann, Bernard Haasdonk, and Mario Ohlberger. Reduced basis approximation for nonlinear parametrized evolution equations based on empirical operator interpolation. SIAM J. Sci. Comput., 34(2):A937-A969, 2012. 


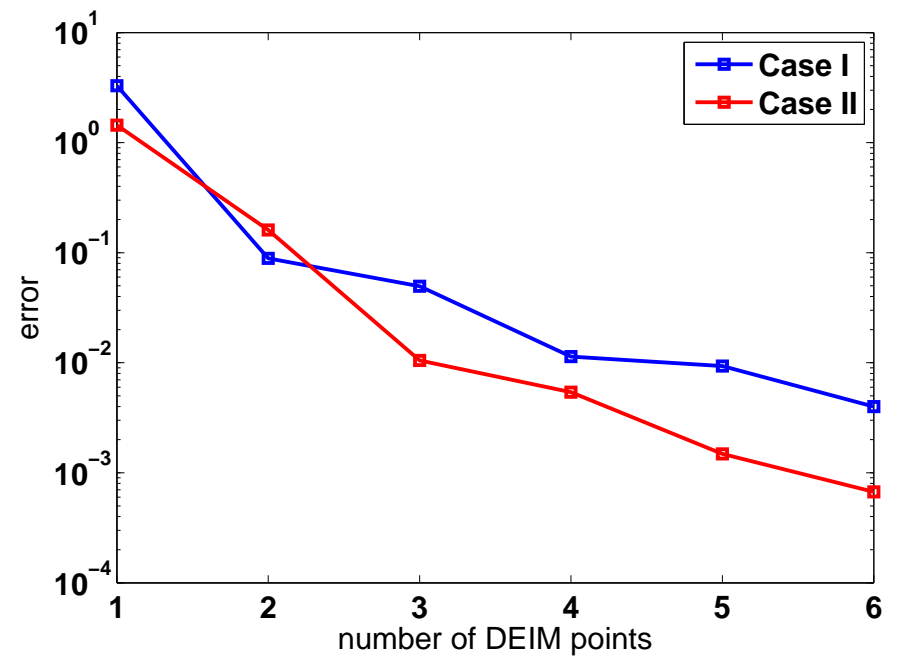

Figure 10: Variations of the relative energy error between the multiscale and multiscale DEIM solutions with the number of DEIM points.

[10] W. E and B. Engquist. Heterogeneous multiscale methods. Comm. Math. Sci., 1(1):87-132, 2003.

[11] Y. Efendiev, J. Galvis, and T. Hou. Generalized multiscale finite element methods. Journal of Computational Physics, 251:116-135, 2013.

[12] Y. Efendiev, J. Galvis, G. Li, and M. Presho. Generalized multiscale finite element methods. oversampling strategies. International Journal for Multiscale Computational Engineering, accepted, 2013.

[13] Y. Efendiev, J. Galvis, M. Moon, R. Lazarov, and M. Sarkis. Generalized multiscale finite element method. symmetric interior penalty coupling. Journal of Computational Physics, accepted, 2013.

[14] Y. Efendiev, J. Galvis, and F. Thomines. A systematic coarse-scale model reduction technique for parameter-dependent flows in highly heterogeneous media and its applications. Multiscale Model. Simul., 10:1317-1343, 2012.

[15] Y. Efendiev, J. Galvis, and X.H. Wu. Multiscale finite element methods for high-contrast problems using local spectral basis functions. Journal of Computational Physics, 230:937955, 2011. 
[16] Y. Efendiev and T. Hou. Multiscale Finite Element Methods: Theory and Applications, volume 4 of Surveys and Tutorials in the Applied Mathematical Sciences. Springer, New York, 2009.

[17] Y. Efendiev, T. Hou, and V. Ginting. Multiscale finite element methods for nonlinear problems and their applications. Comm. Math. Sci., 2:553-589, 2004.

[18] Y. Efendiev and A. Pankov. Numerical homogenization and correctors for nonlinear elliptic equations. SIAM J. Appl. Math., 65(1):43-68, 2004.

[19] Y. Efendiev and A. Pankov. On homogenization of almost periodic nonlinear parabolic operators. Int. J. Evol. Equ., 1(3):203-209, 2005.

[20] Juan Galvis and Yalchin Efendiev. Domain decomposition preconditioners for multiscale flows in high-contrast media. Multiscale Model. Simul., 8(4):1461-1483, 2010.

[21] Juan Galvis and Yalchin Efendiev. Domain decomposition preconditioners for multiscale flows in high contrast media: reduced dimension coarse spaces. Multiscale Model. Simul., 8(5):1621-1644, 2010.

[22] M. Ghommem, M. Presho, V. M. Calo, and Y. Efendiev. Mode decomposition methods for flows in high-contrast porous media. global-local approach. Journal of Computational Physics, Vol. 253., pages 226-238.

[23] M. Hinze and S. Volkwein. Proper orthogonal decomposition surrogate models for nonlinear dynamical systems: error estimates and suboptimal control. In P. Benner, V. Mehrmann, and D.C. Sorensen, editors, Dimension Reduction of Large-Scale Systems, volume 45 of Lecture Notes in Computational Science and Engineering, pages 261-306. Springer Berlin Heidelberg, 2005.

[24] T. Hughes, G. Feijoo, L. Mazzei, and J. Quincy. The variational multiscale method - a paradigm for computational mechanics. Comput. Methods Appl. Mech. Engrg., 166:3-24, 1998.

[25] O. Iliev, R. Lazarov, and J. Willems. Variational multiscale finite element method for flows in highly porous media. Multiscale Model. Simul., 9(4):1350-1372, 2011.

[26] P. Jenny, S.H. Lee, and H. Tchelepi. Multi-scale finite volume method for elliptic problems in subsurface flow simulation. J. Comput. Phys., 187:47-67, 2003. 
[27] M.F. Wheeler, G. Xue, and I. Yotov. A multiscale mortar multipoint flux mixed finite element method. ESAIM Math. Model. Numer. Anal., 46(4):759-796, 2012. 